\title{
Minimally Invasive Approach to Cor Triatriatum Sinister
}

\author{
Mohineesh Kumar ${ }^{1}$ and Francis Shannon ${ }^{1}$ \\ ${ }^{1}$ Beaumont Health
}

July 29, 2020

\begin{abstract}
Cor-triatriatum sinister (CTS) is a rare congenital condition in which the left atrium is divided into two chambers. Patients can present as infants or later in life, which is largely dependent on the size of fenestration between these two chambers. Surgical excision of the membrane is curative. This is traditionally performed through a median sternotomy. Here we report of a case of a 60-year old man who presented to his primary care physician with dyspnea and atrial fibrillation. He was found to have CTS. We performed excision of the left atrial membrane and maze procedure through a left anterolateral mini-thoracotomy with femoral cannulation.
\end{abstract}

\section{Minimally Invasive Approach to Cor Triatriatum Sinister}

Mohineesh Kumar, MD and Francis L. Shannon, MD

Department of Surgery

Section of Cardiovascular Surgery

Oakland University William Beaumont School of Medicine

Royal Oak, Michigan

Corresponding author: Mohineesh Kumar, MD

3601 West Thirteen Mile Road

Royal Oak, Michigan 48073

Phone: 317-459-1058

e-mail: mohineesh.kumar@beaumont.org

\section{Abstract :}

Cor-triatriatum sinister (CTS) is a rare congenital condition in which the left atrium is divided into two chambers. Patients can present as infants or later in life, which is largely dependent on the size of fenestration between these two chambers. Surgical excision of the membrane is curative. This is traditionally performed through a median sternotomy. Here we report of a case of a 60-year old man who presented to his primary care physician with dyspnea and atrial fibrillation. He was found to have CTS. We performed excision of the left atrial membrane and maze procedure through a left anterolateral mini-thoracotomy with femoral cannulation.

\section{Introduction:}

Cor-triatriatum Sinister (CTS) is a rare anomaly in which the left atrium is divided into two distinct chambers separated by a fibromuscular membrane and represents only $0.1 \%$ of all congenital cardiac anomalies. 
${ }^{1}$ Patients develop symptoms secondary to impaired venous return from the pulmonary veins. ${ }^{2}$ The degree of a patient's symptoms is directly related to the size of the left atrial opening and inter-atrial gradient. ${ }^{1}$ Infants can present with heart failure or death in infancy due to complete left atrial obstruction. ${ }^{1}$ In adults, CTS is often asymptomatic due to a large foramen in the membrane and minimal inter-atrial gradient. ${ }^{2}$ CTS can present as a functional mitral stenosis and can present with atrial fibrillation or mitral regurgitation in adults. ${ }^{3}$ Patients presenting later in life are more likely to have lower pressure gradients between the two atria and lower pulmonary capillary wedge pressures. ${ }^{3}$. The most common associated anomalies include atrial septal defect, anomalous pulmonary venous return, tetralogy of Fallot, and bicuspid aortic valve. $^{2}$. Due to the increased general use of imaging modalities, CTS is being more commonly diagnosed. ${ }^{3}$ Incidental asymptomatic CTS may not require treatment. Traditionally CTS has been managed with median sternotomy. Here we report a case of CTS repair done minimally invasively through a right anterolateral mini-thoracotomy.

\section{Case Presentation :}

A 60-year old Caucasian construction worker with no past medical history presented to a primary care physician with a three month history of increasing fatigability, particularly at the end of each day. Electrocardiogram demonstrated atrial fibrillation. Echocardiography (figure 1 ) demonstrated severely enlarged right atrium as well as a severely large left atrium with two atria consistent with Cor-triatriatum sinister. There was a transmembrane gradient of $3 \mathrm{~mm}$ between the two left atria. All four pulmonary veins drained into the upper left atrium. The left atrial appendage also originated from the lower left atrium. The mitral valve was normal in architecture. He had a persistent left superior vena cava and ejection fraction was $60 \%$. CTA was also used to further define anatomy and demonstrated a $1.6 \times 1.5 \mathrm{~cm}$ fenestration in the membrane between the two left atria (figure 2 ).

An anterolateral mini-thoracotomy was made through the right fourth intercostal space. The common femoral artery and vein were cannulated in the right groin for cardiopulmonary bypass. The venous cannula position in the right atrium was confirmed by trans-esophageal echo. The right lung was collapsed with aid of bronchial blocker. This allowed us to visualize the pericardium, which was incised $4 \mathrm{~cm}$ away from and parallel to the phrenic nerve. A purse-string suture was placed in the superior vena cava and $24 \mathrm{Fr}$ single-stage venous cannula was inserted. An anterograde cardioplegia catheter was placed in the ascending aorta. The patient was placed on bypass and a flexible cross clamp occluded the ascending aorta. The heart was arrested with antegrade Custodial cardioplegia. A left atriotomy was made along Waterson's groove. The CTS membrane extended from the fossa ovalis to the superior margin of the left atrial appendage. There was a dime-sized fenestration in the CTS membrane at a right angle to a patent foramen ovale (not seen in pre-operative echo). A mitral lift retractor was used to gain exposure to left atrium. The membrane excision was started at the fenestration and extended circumferentially around the left atrium (figure 3 ). A small cuff of membrane was left at the posterior wall. A running 3-0 prolene was used to close the ostium of the left atrial appendage. A Cox Maze IV left atrial lesion set was done using Atricure bipolar radiofrequency device and cryoprobe. The patent foramen ovale was closed with a 3-0 pledgeted suture. The left atriotomy was closed and the patient was taken off bypass without complication. Post repair TEE showed no mitral valve regurgitation, no left to right shunting and preserved pulmonary venous flow into the left atrium. The rhythm was sinus. The patient's post-operative course was uneventful. Final pathology demonstrated atrial myocardial and fibrous tissue with dystrophic calcification (figure 4 ).

\section{Discussion:}

Our patient had a large dime-sized fenestration in his CTS membrane with minimal (3mm) intra-atrial gradient. Although there was small gradient, there was a significant obstruction of flow. The patient also had a patent foramen ovale, which allowed for left-to-right shunting and allowed for left atrial decompression into the right atrium. This led to a gradual left and right atrial dilation over 60 years and the subsequent development of dyspnea and atrial fibrillation, which is the most common trigger for presenting at a later age. ${ }^{3}$ 
Minimally invasive anterior thoracotomy is a well-described technique for repair and replacement of mitral valves. It provides reduced post-operative pain, decreased infection, improved cosmesis, and reduced length of stay. ${ }^{4}$ For repair of CTS, it provides excellent exposure of the left atrium allowing for complete assessment of the pulmonary veins, left atrial appendage and the CTS membrane. This is only the second reported case of minimally invasive repair of CTS in the literature to our knowledge. ${ }^{5}$ We believe that minimally invasive anterolateral thoracotomy should be the preferred method for treatment of CTS as well as other left atrial and mitral pathology.

\section{Conflicts of Interest :}

There are no conflicts of interest

\section{Author Contributions:}

Concept/Design, Data analysis/interpretation, Drafting article: Mohineesh Kumar

Concept/Design, Data analysis/interpretation, Critical revision and approval of article: Francis Shannon

\section{Bibliography}

1. Jegier W, Gibbons JE and Wiglesworth FW. Cortriatriatum: clinical, hemodynamic and pathological studies surgical correction in early life.Pediatrics . 1963;31:255-67.

2. Nassar PN and Hamdan RH. Cor Triatriatum Sinistrum: Classification and Imaging Modalities. Eur $J$ Cardiovasc Med . 2011;1:84-87.

3. Slight RD, Nzewi OC, Buell R and Mankad PS. Cor-triatriatum sinister presenting in the adult as mitral stenosis: an analysis of factors which may be relevant in late presentation. Heart Lung Circ . 2005;14:8-12.

4. Ward AF, Grossi EA and Galloway AC. Minimally invasive mitral surgery through right mini-thoracotomy under direct vision. J Thorac Dis . 2013;5 Suppl 6:S673-9.

5. Gottschalk BH, Fujii S, Grant A, Iglesias I and Chu MWA. Minimally Invasive Periareolar Approach to Cor Triatriatum Repair.Innovations (Phila) . 2018;13:445-447.

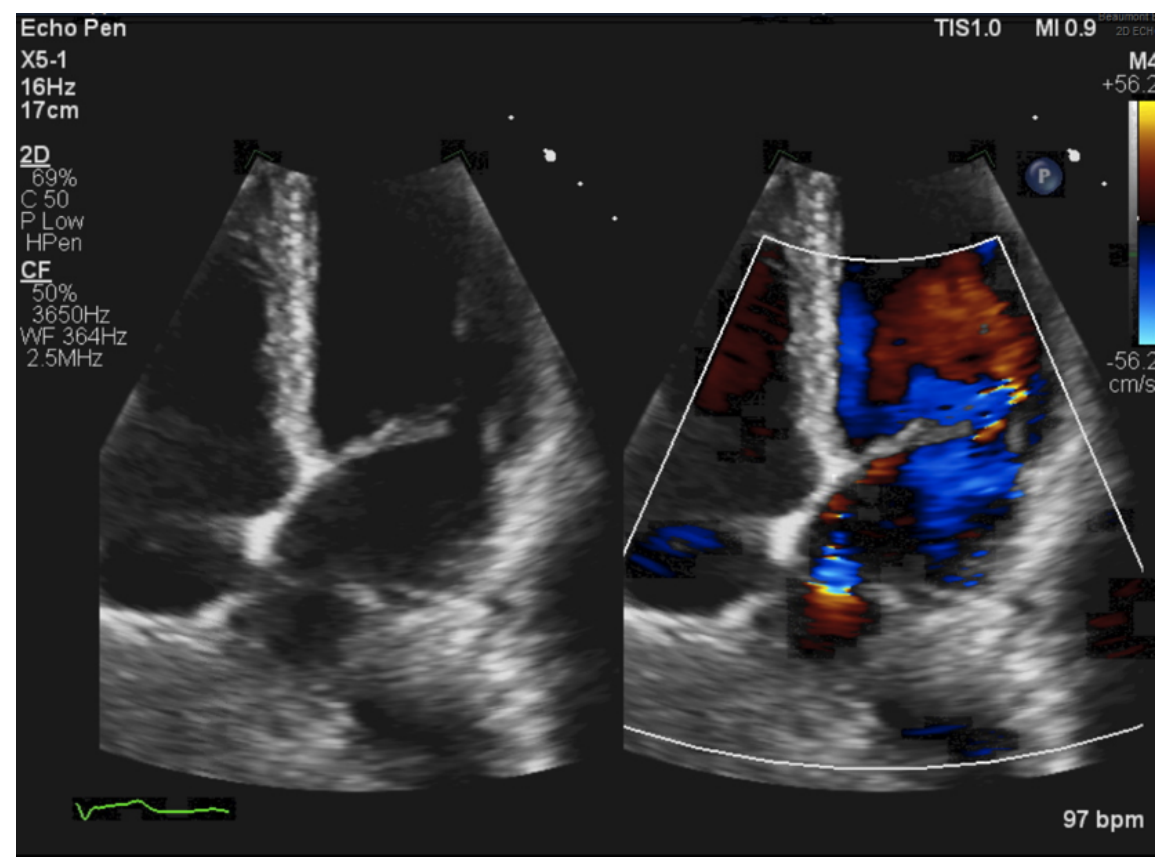


Figure 1: Echocardiography demonstrating cor triatriatum sinister

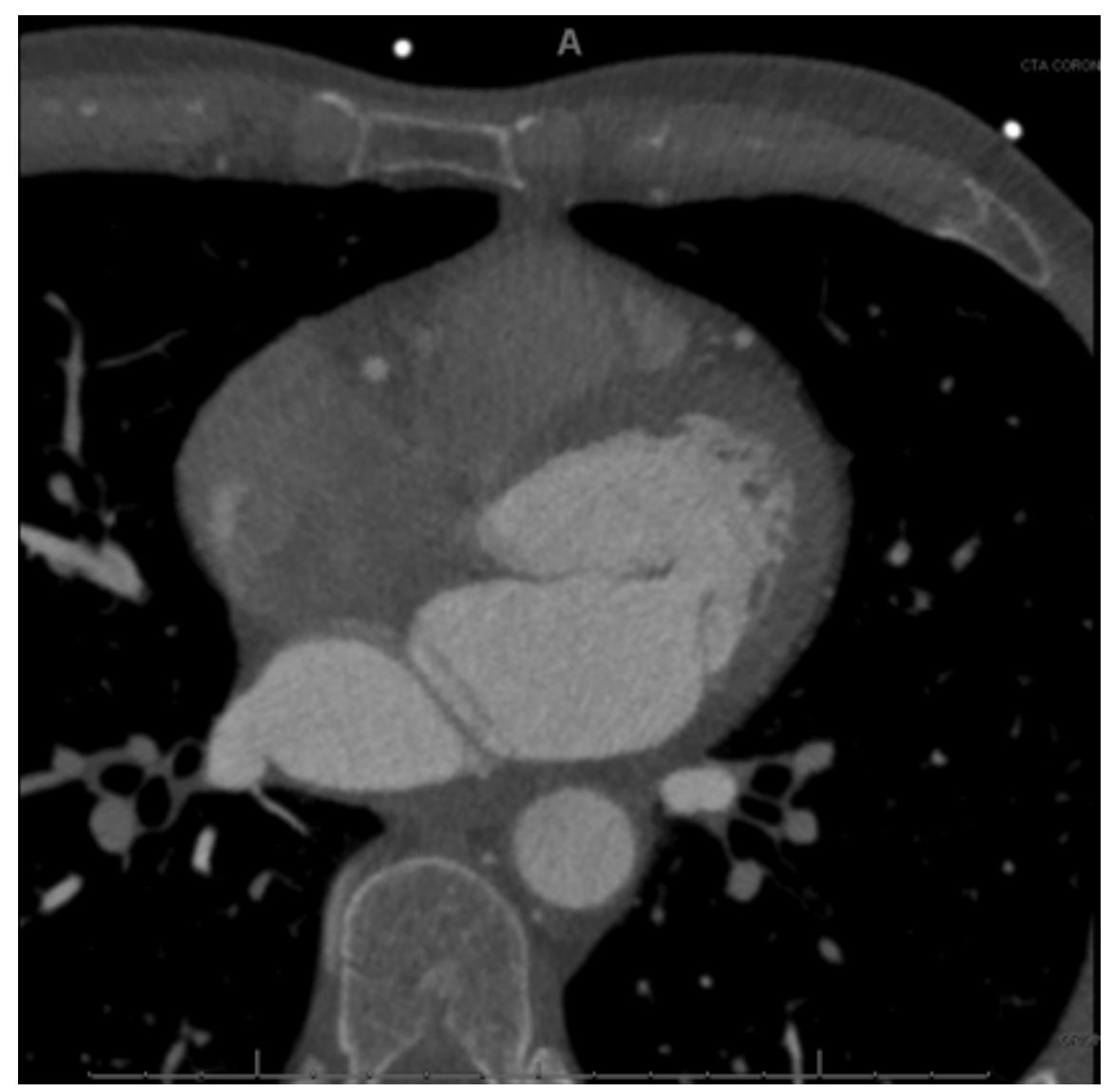

Figure 2: CTA of the heart demonstrating two atria and mitral valve (A), $15 \mathrm{~mm}$ fenestration between the two champers (B), and pulmonary veins draining into left atrium with a thick fibromuscular portion of membrane (C) 


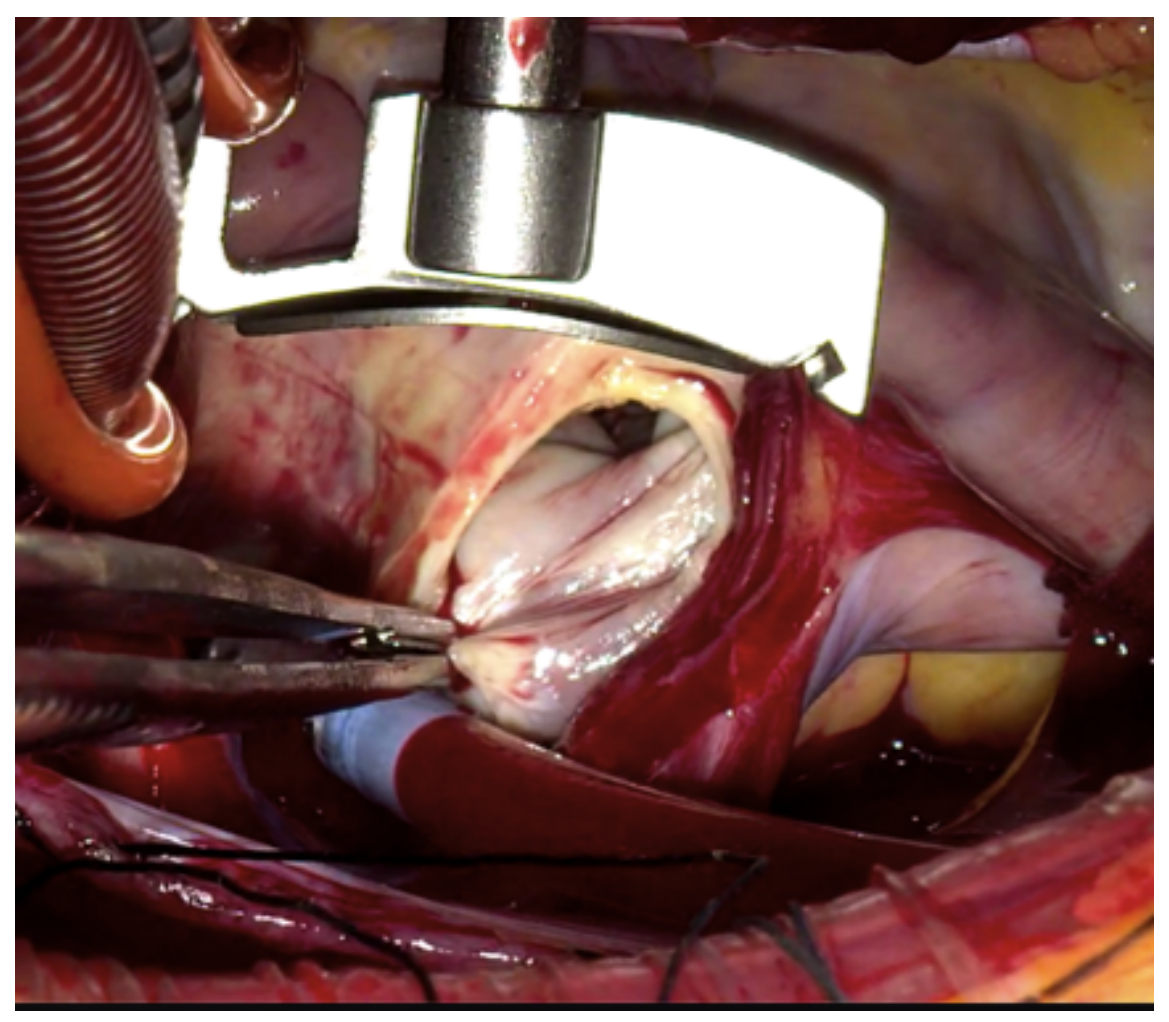

Figure 3: Intra-operative photos after left atriotomy and placement of mitral lift retractor. A drainage catheter was placed in the left atrial appendage. Picture A demonstrates the cor triatriatum in situ. Picture $\mathrm{B}$ shows the left atrium after excision of the membrane. Picture $\mathrm{C}$ shows the closure of the left atrial appendage 


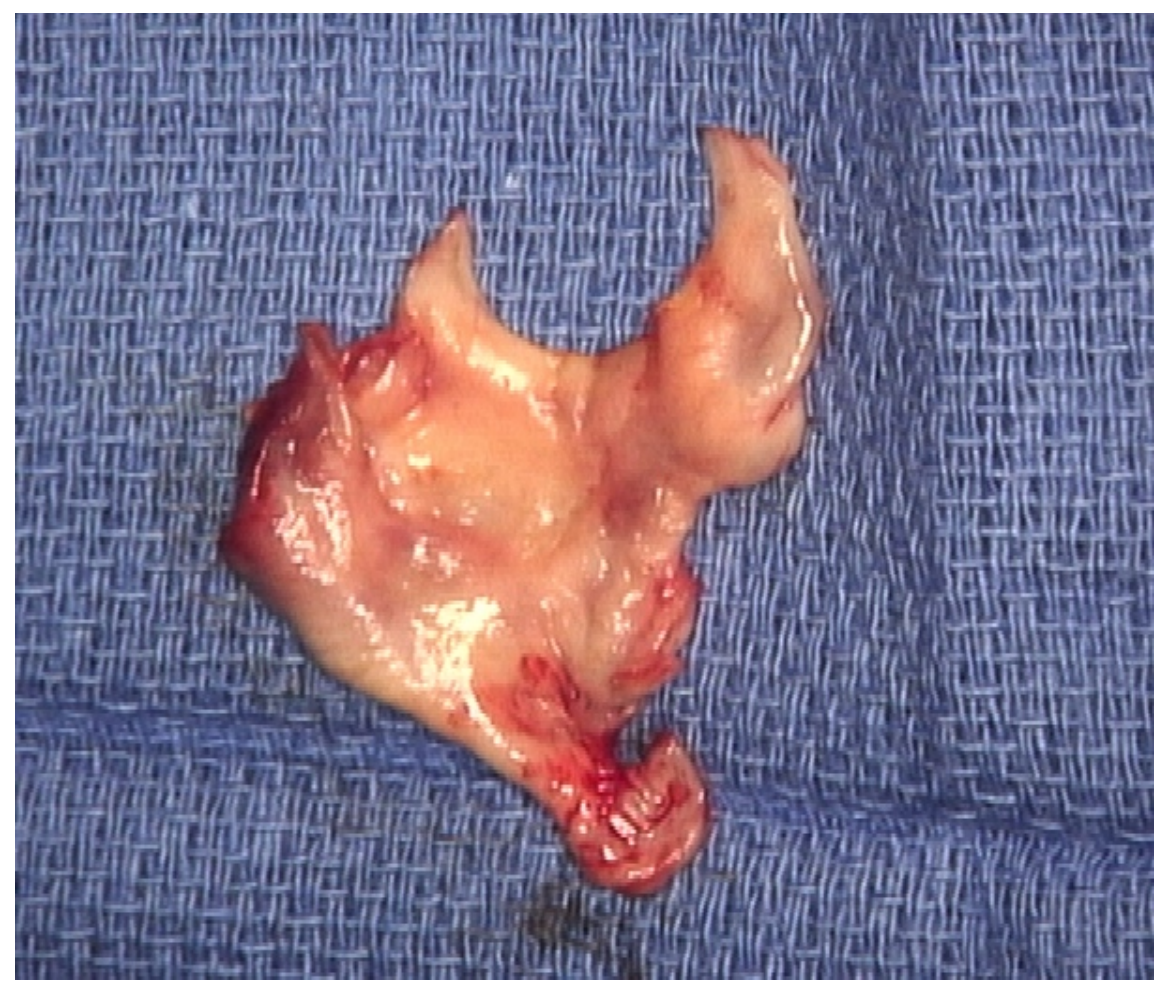

Figure 4: Excised membrane measuring $2.5 \times 2.5 \times 0.4 \mathrm{~cm}$. The dime sized fenestration is the crescent at the superior part of image. 